\title{
LEF1-AS1 contributes to proliferation and invasion through regulating miR-544a/ FOXP1 axis in lung cancer
}

\author{
Ansheng Wang ${ }^{1} \cdot$ Chengling Zhao $^{2} \cdot$ Yuan Gao $^{1}$ (1) Guixin Duan ${ }^{1} \cdot$ Yuming Yang ${ }^{1} \cdot$ Bo Fan $^{1} \cdot$ Xiaojing Wang $^{2}$. \\ Kangwu Wang ${ }^{1}$
}

Received: 30 October 2018 / Accepted: 26 December 2018 / Published online: 8 February 2019

(C) The Author(s) 2019

\begin{abstract}
Long non-coding RNAs (lncRNAs) are increasingly recognized as important regulators in tumor development. This study aims to investigate the potential role oflncRNALEF1-AS1, in the progression of lung cancer. Quantitative real-time PCR (qRT-PCR) and western blot assays showed that LEF1-AS1 was upregulated while miR-544a was downregulated in lung cancer specimens and cells. Overexpression of LEF1-AS1 led to the enhancement of cell proliferation and invasion, revealed by CCK-8 assay and transwell assay. A negative correlation was found between LEF1-AS1 and miR-544a. BLAST analysis and dual-luciferase assay confirmed that FOXP1 is a downstream effector of miR-544a. Therefore, the LEF1-AS1/miR-544a/FOXP1 axis is an important contributor to lung cancer progression. Collectively, our novel data uncovers a new mechanism that governs tumor progression in lung cancer and provides new targets that may be used for disease monitoring and therapeutic intervention of lung cancer.
\end{abstract}

Keywords Lung cancer $\cdot$ Long non-coding RNAs $\cdot$ LEF1-AS1 $\cdot$ LEF1-AS1/miR-544a/FOXP1 axis

\section{Introduction}

Lung cancer is the most common cause of cancer-related deaths in the globe and accounts for an estimated 1.6 million deaths each year [1]. The majority (85\%) of lung cancer patients suffers from non-small cell lung cancer, including adenocarcinomas and squamous cell carcinomas [2]. Due to the high mortality and morbidity of lung cancer, it is imperative to understand the underlying molecular mechanism of lung cancer tumorigenesis to develop new prognostic markers and effective therapeutic strategies [3-5].

Ansheng Wang and Chengling Zhao contributed equally to this work.

Yuan Gao

qawap84@163.com

Guixin Duan

bjquz42@163.com

1 Departments of Thoracic Surgery, The First Affiliated Hospital of Bengbu Medical College, No.287 Changhuai Road, Bengbu City, Anhui Province 233004, People's Republic of China

2 Department of Respiratory Diseases, The First Affiliated Hospital of Bengbu Medical College, No.287 Changhuai Road, Bengbu City, Anhui Province 233004, People's Republic of China
LncRNAs, defined as oligonucleotides with lengths of greater than 200 nucleotides [6, 7], are transcribed by RNA polymerase II and frequently originate from intergenic regions. LncRNAs make up a considerable component of the mammalian transcriptome [6], which do not possess substantial open reading frames and can be spliced, capped and polyadenylated $[8,9]$. Fundamentally, the location, abundance and distribution of IncRNAs throughout the genome provides the organism with an additional method to control the expression of thousands of proteins, by transcriptional and posttranscriptional modifications. Recently, Long non-coding RNAs (IncRNAs) have recently been uncovered in the human genome and found to play a pivotal role in regulating many oncogenic pathways in various cancer types, including those found in lung cancers 6 . Many lncRNAs have been shown to play crucial roles in at least one hallmark of cancer and can behave as either oncogenes or tumor suppressors $[10,11]$.

Human lymphoid enhancer-binding factor 1 antisense RNA 1 (LEF1-AS1) is a newly discovered lncRNA located on the plus strand of chromosome 4 [12]. LEF1-AS1 was previously shown to be upregulated in glioblastoma (GBM) tissues and its dysregulation was postulated to correlate with poor overall survival in patients [13]. Additionally, knockdown of LEF1-AS1 demonstrated tumor-suppressing effects, such as lowering cancer cell proliferation, invasion and 
migration. These findings uncovered a role of LEF1-AS1 as a target oncogene in GBM, but failed to confirm the underlying signaling mechanism. Here, we show that LEF1-AS1 promotes proliferation and invasion in lung cancer by regulating the miR-544a/ FOXP1 axis. These findings may provide a valuable support for LEF1-AS1 used as a potential target for the therapy of lung cancer, as well as establish a foundation for LEF1-AS1 could serves as a novel target for anti-cancer drug in future.

\section{Methods}

\section{Clinical tissue specimens}

A total of 48 pairs of lung cancer tissues and adjacent normal tissue were acquired from The First Affiliated Hospital of Bengbu Medical College between Jan 2012 and Sep 2014. The study protocol was approved by the Ethics Committees of The First Affiliated Hospital of Bengbu Medical College. All patients provided written informed consent. Samples were stored at $-80^{\circ} \mathrm{C}$ until use.

\section{Cell lines and culture}

The normal human lung epithelial cell, BEAS-2B, and human lung cancer cell lines, including H1299, A549, H1975 and SPC-A-1, were purchased from ATCC (Manassas, VA). Cells were cultured in RPMI 1640 medium supplemented with $10 \%$ fetal bovine serum in humidified condition with $95 \%$ air and $5 \% \mathrm{CO}_{2}$ at $37^{\circ} \mathrm{C}$.

\section{Oligonucleotides transfection}

siRNA against LEF1-AS1 (Si-LEF1-AS1), short-hairpin RNA plasmid specific to LEF1-AS1 (sh- LEF1-AS1), miR-544a inhibitor, miR-544a mimics, and their controls were synthesized by GenePharma (Shanghai, China). Oligonucleotide transfection were performed using Lipofectamine 2000 (Invitrogen, Carlsbad, CA) according to the manufacturer's protocol. The sequence of siRNA for LEF1-AS1 and Control: Si-LEF1-AS1, sense 5'-GGCCAAGGAAUUUACUUAUUU-3', antisense 3'UUCCGGUUCCUUAAAUGAAUA-5'; Control: sense: $5^{\prime}$ GGCCGAGGCTCAATGUTTUUU -3', antisense: $5^{\prime}$ UUTTGGUUGGCUAAAGCATUA -3';

\section{BLAST alignment}

NCBI's BLAST suite was used for alignment searches. The top search results with an value $<0.01$ was reported. RNA transcripts were allowed to have multiple exons aligning to different non-contiguous regions of a chromosome. We proceeded our study using miR-544a, a miRNA with a high affinity to LEF1-AS1.

\section{qRT-PCR}

Total RNA were isolated from tissues and cells using the miRNeasy Mini Kit (Qiagen, Valencia, CA, USA) according to the manufacturer's instructions. Quality and concentration of RNA were evaluated with NanoDrop 2000 (Thermo Fisher, Wilmington, DE, USA). cDNA was synthesized by TransScript first-strand cDNA synthesis SuperMix (TransGen, Beijing, China). RT-PCR assay was carried out by ABI prism 7500 sequence detection system (Applied Biosystems Life Technologies) using SYBR green qPCR SuperMix (Applied Biosystems Life Technologies, Foster, CA, USA). The expression of genea was quantified using the $2^{-\Delta \Delta \mathrm{Ct}}$ (cycle threshold), method and the expression levels of miRNA and lncRNA/target gene were normalized by U6 and GADPH, respectively. The primer sequenceswere showed as follows:: LEF1-AS1, forward: 5'-GGGCCCCTTTGTGTGACTAA-3'; reverse, 5'ACCTGCGCTAAGAACTGAGG-3'; miR-544a, forward: 5'TAAAAGCTGGCAACTGTCTAA-3', reverse, 5'ATTAGTAGGAAATTGCTGCAG-3'; GAPDH, forward, 5'TCGACAGTCA GCCGCATCTTCTTT-3', reverse, 5'-ACCA AATCCGTTGACTCCGACCTT-3'.

\section{Luciferase reporter assay}

LEF1-AS1 cDNA fragment that encompassed microRNA binding sites was inserted into the pmirGLO plasmids (Promega, Madison, WI, USA). Mutant LEF1-AS 1 (pmirGLO- LEF1-AS1-MUT) generated by site-directed mutagenesis PCR with platinum pfx DNA polymerase was which served as the negative control. Target miR-544a mimics or miR-NC mimics and luciferase reporter plasmids and were cotransfected into cells using Lipofectamine 2000. At 48 hafter transfection, relative luciferase activity was measured in a luminometer by Dual-Luciferase Reporter Assay System (Promega).

\section{Cell proliferation assay}

Cell Counting Kit-8 (CCK- 8; Dojindo, JPN) was used to assess cell proliferation. A549 and H1299 cells transfected with si-NC, si-LEF1-AS1, miR-544a inhibitor or si- LEF1-AS1+ miR-544a inhibitor were collected and seeded into 96-well plates. After 24, 4872 or $96 \mathrm{~h}, 10 \mu \mathrm{l}$ of CCK-8 assay reagent was added to each well. After incubation for $2 \mathrm{~h}$, DMSO was added and the absorbance was measured using an enzyme immunoassay analyser (Bio-rad, Hercules, CA, USA). 


\section{Cell migration and invasion assay}

Wound healing assay and transwell assay were performed to measure the migration and invasion ability of breast cancer cells respectively. For wound healing assay, when A549 CSC and H1299 CSC cells were cultured to 90\% confluence in 96well plates, the medium was removed and a gap was made by enforcing the sterile pipette tip on the monolayer cells. The width of the wound gap at $24 \mathrm{~h}$ was acquired and normalized to initial distance at $0 \mathrm{~h}$. Migration rate was calculated using the following formula: migration rate $=$ migration distance/ original distance. For transwell assay, A549 CSC and H1299 CSC cells were suspended in $200 \mathrm{ml}$ serum-free DMEM and seeded in chambers ( $8 \mathrm{~mm}, \mathrm{BD}$ Biosciences) coated with BD BioCoat Matrigel. After incubation, the non-invaded cells on the upper membrane surface were removed with a cotton tip. The cells on membrane were fixed and stained by violet crystalline.

\section{Western blot analysis}

The total protein was extracted using the RIPA buffer (Sigma-Aldrich, St. Louis, MO) supplemented with protease inhibitors cocktail (Roche, Diagnostics, Mannheim, Germany). Protein concentration was measured using BCA assay. Proteins were separated by SDS-PAGE, followed by being transferred to PVDF membrane (Millipore, Bedford, MA). After blocked with 5\% nonfat milk, the membrane was incubated with the primary antibodies, including anti-FOXP1 (1:1000, Abcam, Cambridge, MA), anti-GADPH (1:1000, Abcam) d. After washing with TBST, PVDF membrane was incubated with HRP-conjugated goat anti-rabbit IgG (Abcam) at room temperature for $2 \mathrm{~h}$. Finally, the films were developed using ECL detection kit (Beyotime Biotechnology, Shanghai, China).

\section{Lentivirus construction and infection}

Construction of a lentiviral vector expressing LEF1-AS1shRNA was performed by Shanghai Genechem. LEF1-AS1shRNA was inserted into $\mathrm{pFU}-\mathrm{GW}-\mathrm{RNAi}$ vector carrying the green fluorescent protein (GFP) reporter driven by the U6 promoter. A549 cells were seeded into 6-well plates with $2 \times 10$ [5] cells per well. After $12 \mathrm{~h}$, A549 cells were infected with Lv-shRNA-NC or Lv-shRNA- LEF1-AS1 at $10 \mathrm{MOI}$, respectively. Culture medium was changed at $12 \mathrm{~h}$ after infection.

\section{Animal experiments}

All animal experiments were performed according to protocols and approved by the Institutional Animal Care and Use
Committee of The First Affiliated Hospital of Bengbu Medical College. Briefly, $1 \times 10$ [6] A549 cells infected with lentivirus carrying sh- LEF1-AS1 or sh-NC were subcutaneously injected. Tumor size was measured by a caliper every 3 days. Tumor volume was calculated using the following formula: volume $=0.5 \times$ length $\times$ width [2] .

\section{Immunohistochemical staining}

Tumor tissue were sectioned at the thickness of $5 \mu \mathrm{m}$ and embedded in paraffin. To perform immunohistochemical staining, tissues were dewaxed and rehydrated in graded concentrations of xylene/alcohol. Antigen retrieval was performed in citrate buffer (pH 6.0) and heating at $121{ }^{\circ} \mathrm{C}$. Sections were then blocked in goat serum (Boster, Wuhan, China) for $30 \mathrm{~min}$ at room temperature. Ki67 antibody (Bioss Antibodies, Inc., 1:200) was used to incubate the sections overnight at $4{ }^{\circ} \mathrm{C}$. For TUNEL assay, Colorimetric TUNEL Apoptosis Assay Kit (Beyotime, Shanghai, China) was used to incubate the sections at $37{ }^{\circ} \mathrm{C}$ for $60 \mathrm{~min}$. Following, Polink-1 HRP DAB Detection System One-step polymer detection system (ZSGB-BIO, Beijing, China) were added to the section and incubated for $20 \mathrm{~min}$ at room temperature. Hematoxylin was lastly used to stain the nucleus.

\section{Statistical analysis}

All the statistical data are presented as the means \pm S.D. Two-tailed Student's t test or one-way ANOVA followed by the LSD post hoc test was performed for comparisons between groups. Expression correlation assays were analyzed using Pearson's coefficient correlation. Differences in patient survival were performed using the Kaplan-Meier method and analyzed by log-rank test. A value of $P<0.05$ was considered to be statistically significant.

\section{Results}

\section{LEF1-AS1 upregulation in lung cancer is associated with the poor survival of patients}

To explore the role of LEF1-AS1 in lung cancer, qRT-PCR analysis was first performed to detect the expression of LEF1AS1 in lung cancer specimens and adjacent normal tissue from patients $(N=48)$. We found thats, LEF1-AS1 expression was significantly higher in tumor tissues comparing with the adjacent tissues $(P<0.05$, Fig. 1a). Next, we divided the patients into two groups based on the LEF1-AS1 expression, using the average LEF1-AS1 level as the threshold (Fig. 1b). Survival analysis of showed that the overall survival of patients with high LEF1-AS1 expression was much poorer than those with low LEF1-AS1 expression (Fig. 1c), suggesting 
Fig. 1 LEF1-AS1 upregulation is associated with lung cancer aggressiveness. a, qRT-PCR analysis of LEF1-AS1 expression, suggesting a higher LEF1AS1 expression in tumor tissue, compared to normal tissue from lung cancer patients (stage I and II) $(N=48) * p<0.05$. b, Grouping of patients according to LEF1-AS1 expression. Highexpression patients or lowexpression patients were grouped based on average LEF1-AS1 expression. c, Overall survival curve of patients with high or low LEF1-AS1 expression. * $p<0.05$ a

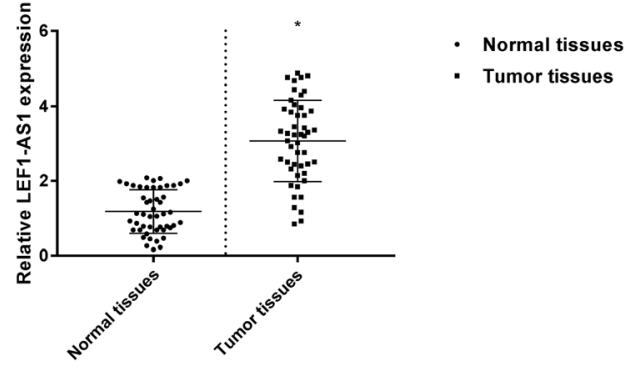

b

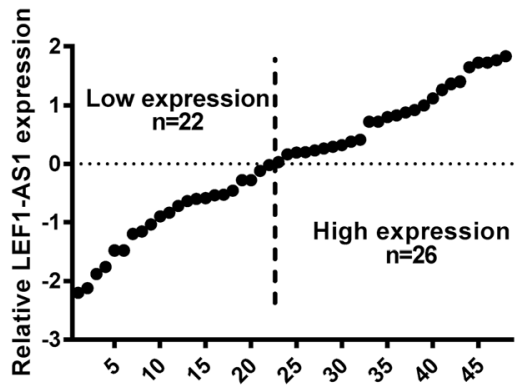

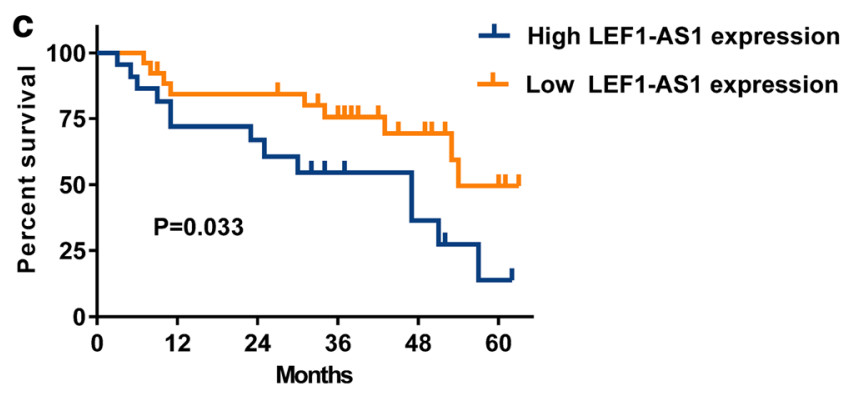

a
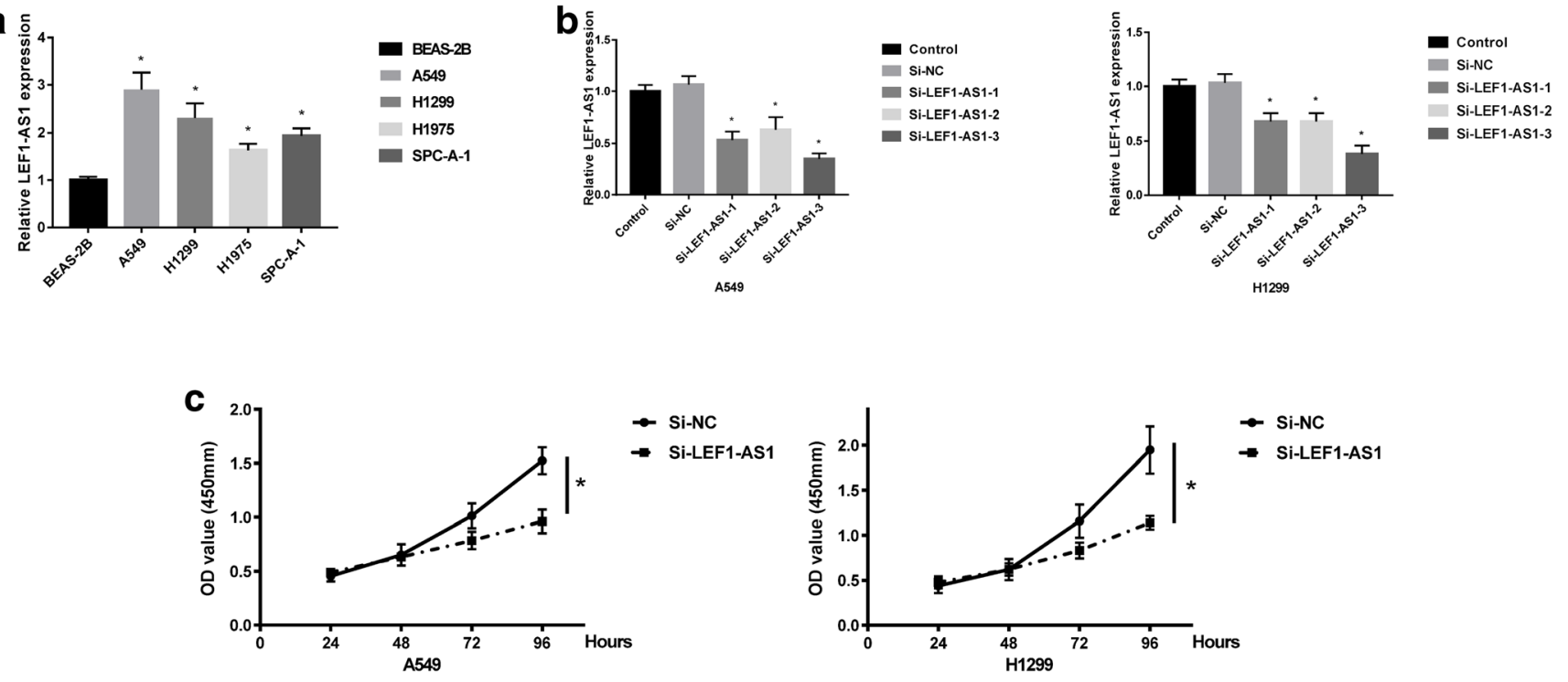

d
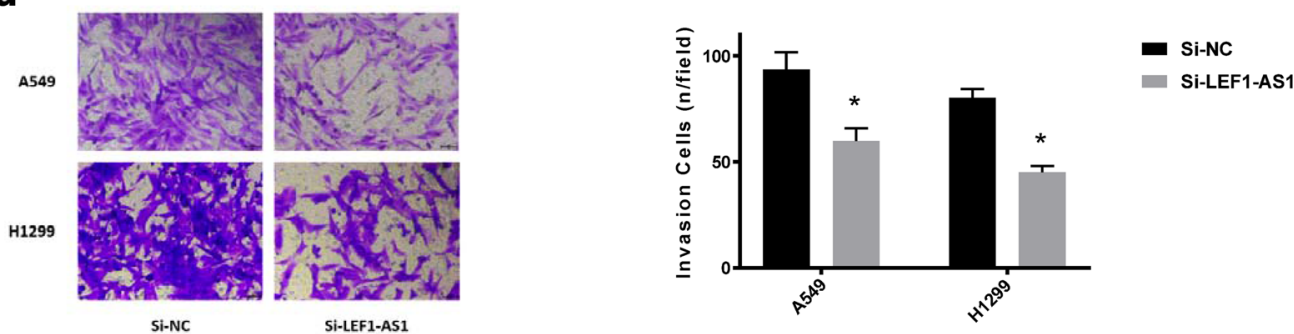

Fig. 2 LEF1-AS1 promotes lung cancer proliferation and invasion. a, Comparison of LEF1-AS1 expression in lung cnacer cells using qRTPCR analysis. b, Change of LEF1-AS1 levels after A549 cells or H1299 cells were transfected with three si-LEF1-AS1s (si-LEF1-AS11, si-LEF1-AS1-2 and si-LEF1-AS1-3). c. Proliferation assay of A549

cells or H1299 cells after si-LEF1-AS-31 transfection. Cells transfected with si-NC were used as a control. d, Representative staining images and quantitative analysis of invaded cells in the lower chamber of transwell assay, comparing the invasive ability of A549 or H1299 cells transfected with si-NC or si-LEF1-AS1. $* P<0.05$ 
that high LEF1-AS1 was associated with lung cancermalignancy and poor survival of patients.

\section{LEF1-AS1 promotes lung cancer proliferation and invasion}

To further confirm the role of LEF1-AS1 in lung cancer, we analyzed the expression of LEF1-AS1in lung cancer A549, H1299, H1975 and SPC-A1 cells, with BEAS-2B cells as the control. Consistent with the upregulation of LEF1-AS1 in lung cancer tissues, LEF1-AS1 expression was also significantly increased in the four tumor cells, compared to BEAS2B cells (Fig. 2a). Especially, A549 and H1299 cells demonstrated the most prominent LEF1-AS1 upregulation, thus which were selected for subsequent studies. LEF1-AS1 silencing was achieved by transfecting three si-LEF1-AS1s into A549 and H1299 cells. Obviously, all three siRNAs induced pronounced LEF1-AS1 downregulation. Since si-LEF1-AS13 showed the most marked LEF1-AS1 downregulation, siLEF1-AS1-3 was used as the lead siRNA to suppress LEF1-AS1 expression in lung cancer cells (Fig. 2b). In A549 and H1299 cells, si-LEF1-AS1 transfection significantly attenuated cell proliferation (Fig. 2c) and invasion (Fig. 2d). These data confirmed the tumor-promoting role of LEF1-AS1 in lung cancer.

a

$$
\begin{array}{r}
\text { LEF-AS1 WT 5'- CATAGGGAGATTAATGCAGAAC - 3' } \\
\text { |||||||| } \\
\text { miR-544a } \quad \text { 3'- CTTGAACGATTTTTACGTCTTA - 5' }
\end{array}
$$

LEF-AS1 MUT 5' - CATAGGGAGATTTTACGTCTTC - 3'

\section{miR-544a is the target of LEF1-AS1}

To clarify the mechanism of LEF1-AS1 in lung cancer regulation, we performed BLAST analysis and identified a binding site between LEF1-AS1 and miR-544a (Fig. 3a). Further, a mutated LEF1-AS1 sequence was designed to explore the specificity of the interaction between LEF1-AS1-WT and miR-544a (Fig. 3a). As shown in Fig. 3b, the result of dualluciferase assay, indicated that miR-544a mimic led to a marked attenuation of luciferase activity induced by LEF1AS1-WT but not LEF1-AS1-MUT (Fig. 3b). Similarly, transfection of miR-544a mimic also resulted in a remarkable downregulation of LEF1-AS1 in A549 and H1299 cells, while miR-544a inhibitor exerted the opposing effects (Fig. 3c). Consistently, transfection of si-LEF1-AS1 significantly upregulated miR-544a expression, while the negative control showed no such effects (Fig. 3d). These data suggested the direct interaction between miR-544a and LEF1-AS1.

\section{Regulation of lung cancer cells by LEF1-AS1 was mediated by miR-544a}

Next, we examined the function of miR-544a in mediating the tumor-promoting effects of LEF1-AS1 in lung cancer cells. It was found that the cells transfected with si-LEF1-AS1 presented a well-markedattenuation of cell proliferation
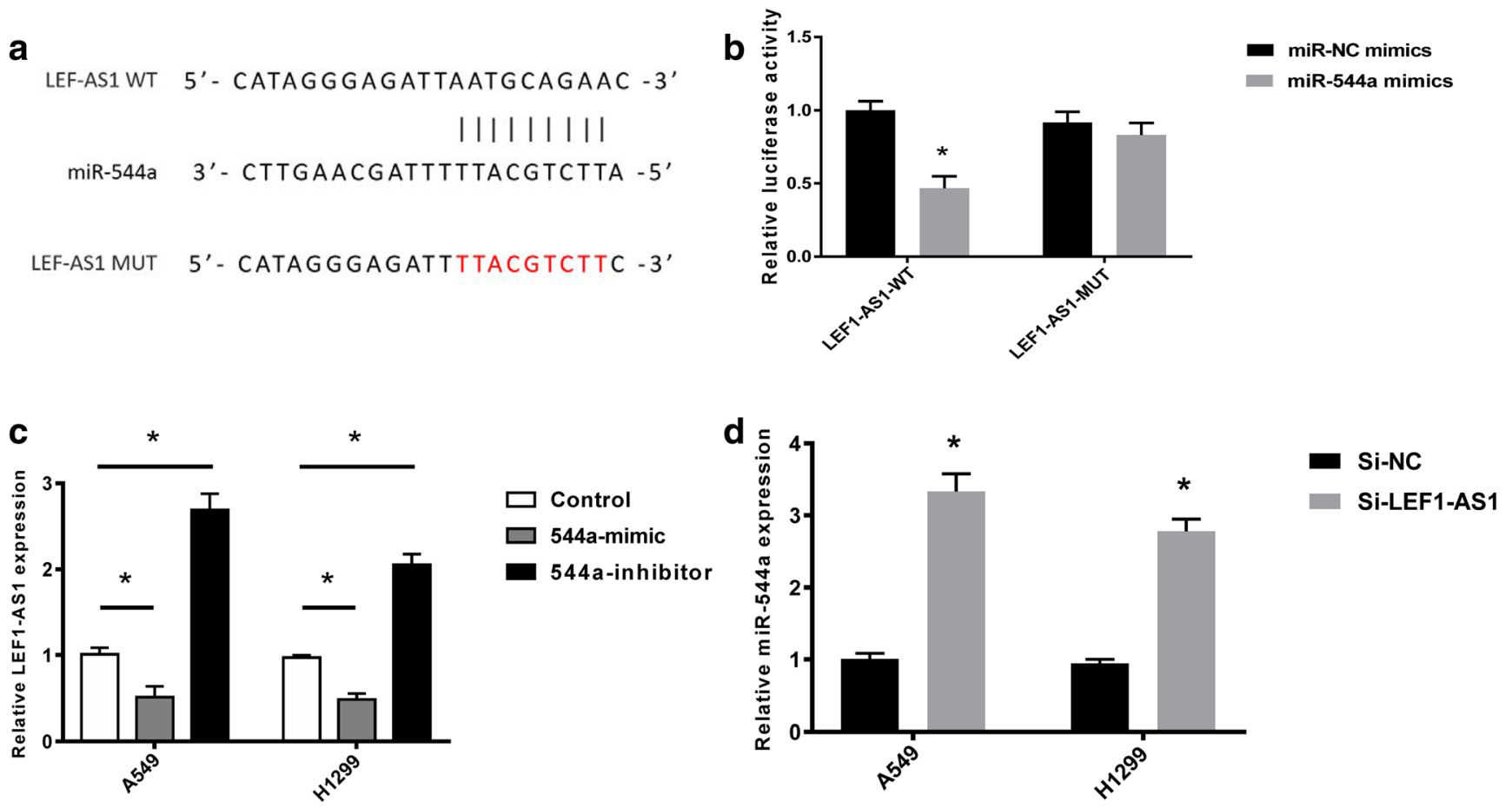

Fig. 3 miR-544a is the target of LEF1-AS1. a, BLAST analysis, which identified a binding site between wild-type LEF1-AS1 (LEF1-AS1-WT) and miR-544a. LEF1-AS1 mutant (LEF1-AS1-MUT) sequence was designed for further luciferase study. $\mathbf{b}$, Cells were transfected indicated vectors and dual-luciferase assay was used to study the relationship

between miR-NC/miR-544a and LEF1-AS1-WT or LEF1-AS1-MUT. c, Analysis of LEF1-AS1 expression using qRT-PCR in A549 and H1299 after 544a mimic or 544a-inhibitor transfection. d, analysis of 544a expression in A549 or H1299 cells after si-LEF1-AS1 or si-NC infection. $* \mathrm{p}<0.05$ 
(Fig. 4a), invasion (Fig. 4b) and migration (Fig. 4c). whereas the opposite effects were observed when miR-544a was knocked down. Notably, co-transfection with si-LEF1-AS1 and miR-544a inhibitor failed to alter the cell proliferation, invasion, and migration compared to cells without transfection. Moreover, a known effector of miR-544a, FOXP1, exhibited a negative correlation to LEF1-AS1 expression (Fig. 4d). Therefore, miR-544a plays an important role in mediating the effects of si-LEF1-AS1 in lung cancer.

\section{LEF1-AS1 inhibition attenuates lung cancer xenograft growth in mice}

To evaluate the anti-tumor effects of LEF1-AS1 silencing in vivo, we established A549 cells stably expressing shcontrol or sh-LEF1-AS1 We found that tumors with shLEF1-AS1 demonstrated significantly smaller sizes (Fig. 5a), as well as decreased Ki-67 expression comparing with the control (Fig. 5b).,Meanwhile, the tumors transfected with sh-LEF1-AS1 also exhibited an we obsversed obvious reduction of LEF1-AS1 expression and a prominent increase
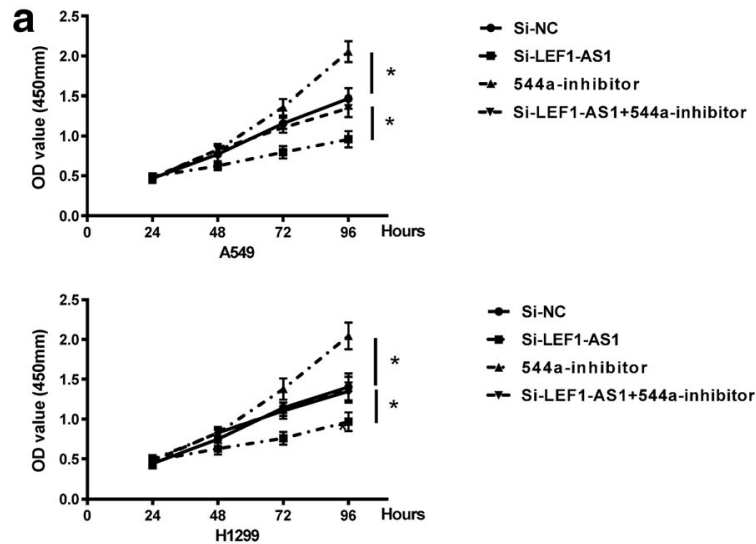

$\rightarrow$ Si-NC

$\rightarrow$ Si-LEF1-AS1

-2 544a-inhibitor

$\rightarrow-$ Si-LEF1-AS1+544a-inhibito

b

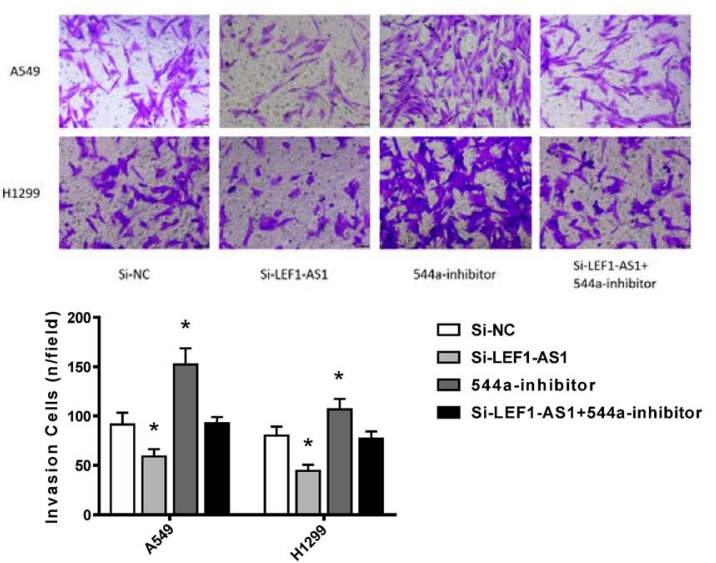

Fig. 4 Regulation of lung cancer cells by LEF1-AS1 was mediated by miR-544a. CCK-8 proliferation assay (a), transwell assay (b) and scratch wound assay (c) n cells transfected by si-NC, si-LEF1-AS1, 544a of miR-544a expression (Fig. 5c and d). Additionally, FOXP1 was downregulated in tumors with LEF1-AS1 silencing (Fig. 5e). These data validated that LEF1-AS1 silencing may be an effective strategy in inhibiting lung cancer growth.

\section{Discussions}

In the present study, we strived to unravel the role of LEF1-AS1 in lung cancer. A previous study indicated that LEF1-AS1 acts as an oncogene in GMA but failed to identify the underlying mechanism promoting malignancy [13]. Our data in a lung cancer model reinforces the oncogenic role of LEF1-AS1 since qRT-PCR analysis revealed higher LEF1-AS1 expression in tumor tissue, compared to paired normal tissue. These differences were also confirmed in cell lines, where LEF1-AS1 was found to be significantly upregulated in a number of lung cancer cell lines, compared to a normal bronchial epithelial cell line. Several studies have recently demonstrated the link between dysregulated lncRNA expression and cancer tumorigenesis, treatment resistance, and metastasis [14-16]. The

C
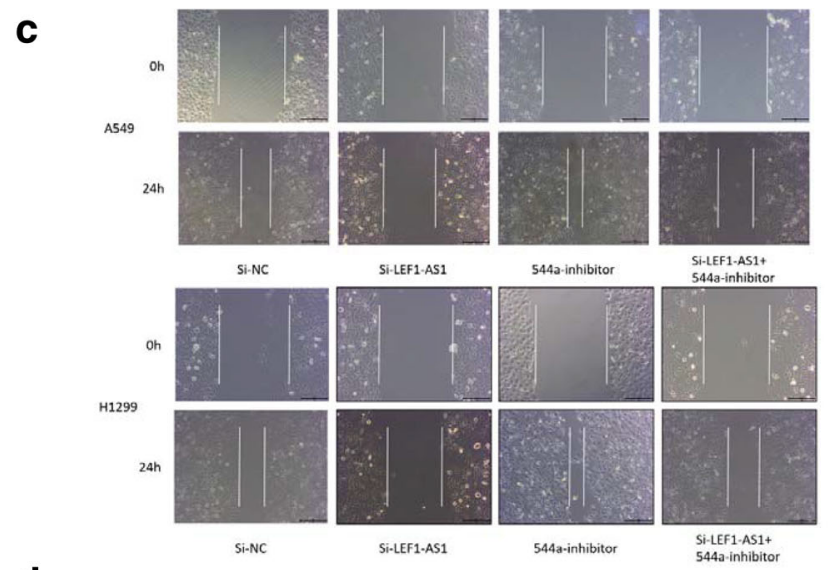

Si-LEF1-AS1

544a-inhibitor

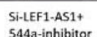

d
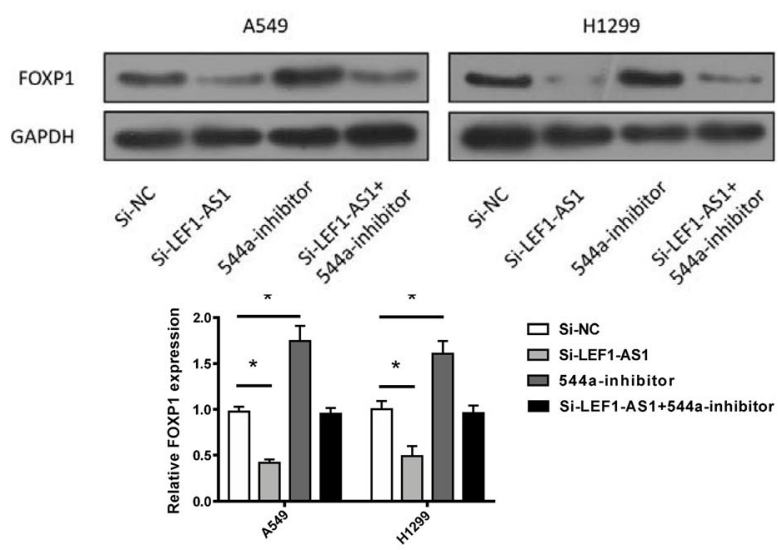

$\square$ Si-NC

$\square$ Si-LEF1-AS1

口 544 a-inhibitor

- Si-LEF1-AS1+544a-inhibitor

inhibitor, or si-LEF1-AS1 + 544a inhibitor. d, western blot analysis of FOXP1 levels in cells transfected by si-NC, si-LEF1-AS1, 544a inhibitor, or si-LEF1-AS1 + 544a inhibitor. $* \mathrm{p}<0.05$ 


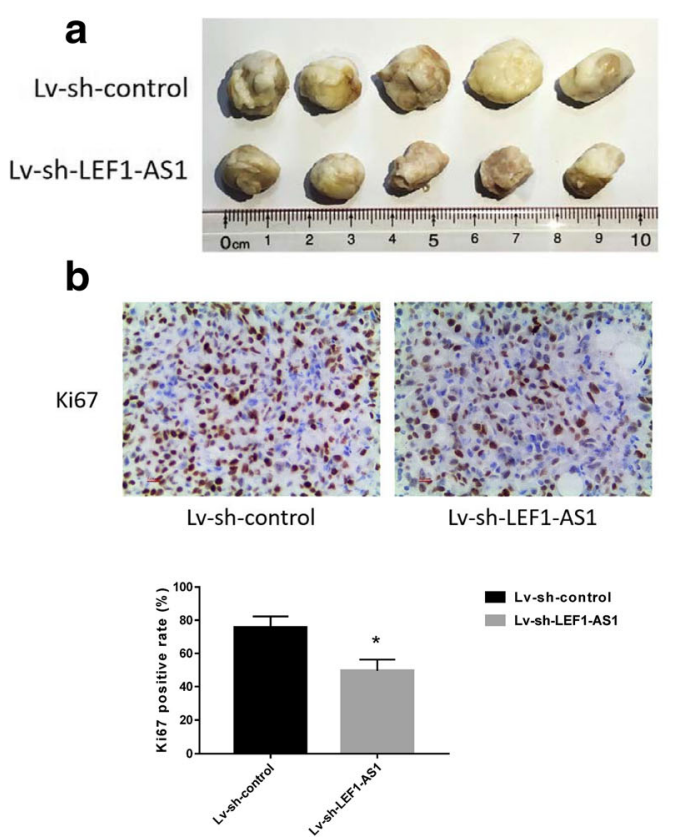

Fig. 5 LEF1-AS1 inhibition attenuates lung cancer xenograft growth in mice. a, Visual examination of tumors initiated from A549 cells infected with lv-sh-control or lv-sh-LEF1-AS1. b, Representative images

\section{C}

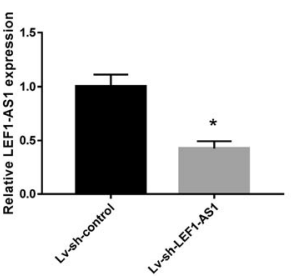

d

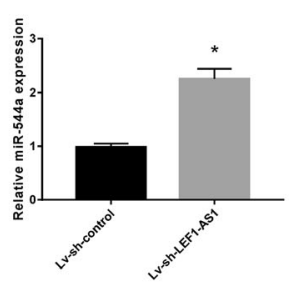

e
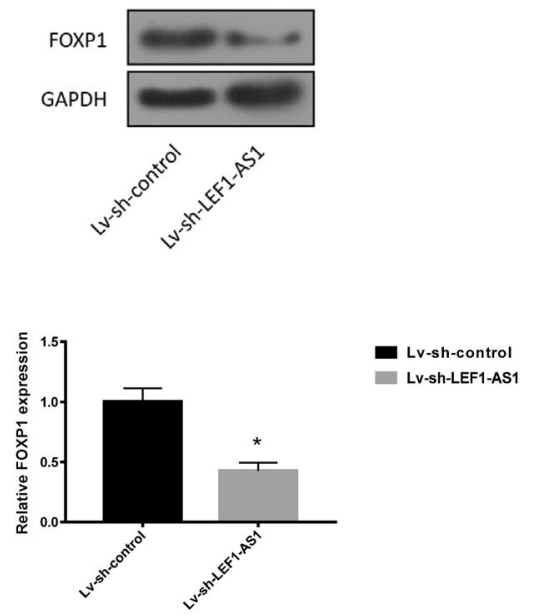

interactions between lncRNAs and macromolecules can influence multiple regulatory mechanisms of cancer either through epigenetic regulation of protein expression or direct dysregulation of lncRNAs [17]. It has been postulated that the deregulation of lncRNAs influences normal regulation of the eukaryotic genome to confer a growth advantage to cancer cells, leading to sustained and uninhibited tumor growth [18]. In support of this hypothesis, IncRNA AB073614 was shown to induce tumor progression and was associated with poor prognosis by regulating ERK1/2 and Akt signaling in ovarian cancer [19]. Additionally, lncRNA CRNDE was shown to impart prooncogenic abilities in gliomas by modulating mTOR signaling [20]. Several studies also indicate that lncRNAs may serve as sensitive biomarkers of specific cancer subtypes based on their cellular specificities [21, 22].

Silencing LEF1-AS1 in lung tumor cells significantly attenuated cell proliferation and invasion. After confirming the oncogenic role of LEF1-AS1, we aimed to elucidate its binding partners. Initially, BLAST analysis uncovered miR-544a as a binding partner of LEF1-AS1. MiR-544ais already a wellknown inducer of epithelial-mesenchymal transition in cancer [23]. We found silencing LEF1-AS1 resulted in upregulation of miR-544a, suggesting a direct interaction between miR544a and LEF1-AS1. This relationship was further confirmed by the negative correlation between LEF1-AS1 expression and FOXP1 expression, which is a well-known effector of miRNAs [24], Silencing LEF1-AS1 also significantly increased miR-544a expression, downregulated FOXP1 expression, lower tumor size and $\mathrm{Ki}-67$ expression. and quantitative analysis of $\mathrm{Ki}-67$ staining of tumor sections. Expression of LEF1-AS1 (c) and miR-544a (d) were quantified by qRT-PCR. e, western blot analysis of FOXP1 expression in tumors. $* p<0.05$

Our findings support the previous in vivo studies showing that tumors with LEF1-AS1 knockdown cells grow more slowly compared to controlsvia modulating ERK1/2 and Akt/mTOR signaling [13]. .Besides, miR-544 has been also found to interrupt adaptive responses to hypoxia via ATMmTOR signaling [25]. LncRNA-based therapeutics are novel anti-cancer strategies that have increasingly garnered attention [26]. Understanding the underlying molecular mechanism of IncRNA therapy is of paramount importance. Through the modulation of LEF1-AS1 expression and possibly other lncRNAs, a new treatment can be formed in the fight against lung cancer and other cancers [27].

\section{Conclusions}

In summary, the present study reveals that LEF1-AS1 is upregulated in lung cancer cell lines and tumors, which plays a positive regulatory role in lung cancer proliferation and invasion. Besides, there was a negative correlation between LEF1AS1 and miR-544a, and FOXP1 is a downstream effector of miR-544a. Fundamentally, the LEF1-AS1/miR-544a/FOXP1 axis is an important contributor to lung cancer progression and that disrupting these signaling pathways could provide a novel mechanism for treating lung cancer.

Funding The work was supported by National Natural Science Foundation of China (81772493). 


\section{Compliance with ethical standards}

Conflict of interests Author Ansheng Wang declares that he has no conflict of interests. Author Chengling Zhao declares that he has no conflict of interests. Author Yuan Gao declares that he has no conflict of interests. Author Guixin Duan declares that he has no conflict of interests. Author Yuming Yang declares that he has no conflict of interests. Author Bo Fan declares that he has no conflict of interests. Author Xiaojing Wang declares that she has no conflict of interests. Author Kangwu Wang declares that he has no conflict of interests.

Ethical approval All procedures performed in studies involving human participants were in accordance with the ethical standards of the institutional research committee and with the 1964 Helsinki declaration and its later amendments or comparable ethical standards. All applicable institutional guidelines for the care and use of animals were followed.

Open Access This article is distributed under the terms of the Creative Commons Attribution 4.0 International License (http:// creativecommons.org/licenses/by/4.0/), which permits unrestricted use, distribution, and reproduction in any medium, provided you give appropriate credit to the original author(s) and the source, provide a link to the Creative Commons license, and indicate if changes were made.

Publisher's note Springer Nature remains neutral with regard to jurisdictional claims in published maps and institutional affiliations.

\section{References}

1. Torre LA, Bray F, Siegel RL, Ferlay J, Lortet-Tieulent J, Jemal A (2015) Global cancer statistics, 2012. CA Cancer J Clin 65(2):87-108

2. Molina JR, Yang P, Cassivi SD, Schild SE, Adjei AA (2008) Nonsmall cell lung cancer: epidemiology, risk factors, treatment, and survivorship. Mayo Clin Proc 83(5):584-594

3. Roselli M, Mariotti S, Ferroni P, Laudisi A, Mineo D, Pompeo E, Ambrogi V, Mineo TC (2006) Postsurgical chemotherapy in stage IB nonsmall cell lung cancer: long-term survival in a randomized study. Int J Cancer 119(4):955-960

4. Douillard JY, Rosell R, De Lena M et al (2006) Adjuvant vinorelbine plus cisplatin versus observation in patients with completely resected stage IB-IIIA non-small-cell lung cancer (adjuvant Navelbine international Trialist association [ANITA]): a randomised controlled trial. Lancet Oncol 7(9):719-727

5. Winton T, Livingston R, Johnson D, Rigas J, Johnston M, Butts C, Cormier Y, Goss G, Inculet R, Vallieres E, Fry W, Bethune D, Ayoub J, Ding K, Seymour L, Graham B, Tsao MS, Gandara D, Kesler K, Demmy T, Shepherd F (2005) Vinorelbine plus cisplatin vs. observation in resected non-small-cell lung cancer. N Engl J Med 352(25):2589-2597

6. Iyer MK, Niknafs YS, Malik R, Singhal U, Sahu A, Hosono Y, Barrette TR, Prensner JR, Evans JR, Zhao S, Poliakov A, Cao X, Dhanasekaran SM, Wu YM, Robinson DR, Beer DG, Feng FY, Iyer HK, Chinnaiyan AM (2015) The landscape of long noncoding RNAs in the human transcriptome. Nat Genet 47:199-208

7. Derrien T, Johnson R, Bussotti G, Tanzer A, Djebali S, Tilgner H, Guernec G, Martin D, Merkel A, Knowles DG, Lagarde J, Veeravalli L, Ruan X, Ruan Y, Lassmann T, Carninci P, Brown JB, Lipovich L, Gonzalez JM, Thomas M, Davis CA, Shiekhattar R, Gingeras TR, Hubbard TJ, Notredame C, Harrow J, Guigo R (2012) The GENCODE v7 catalog of human long noncoding RNAs: analysis of their gene structure, evolution, and expression. Genome Res 22(9):1775-1789
8. Rinn JL, Chang HY (2012) Genome regulation by long noncoding RNAs. Annu Rev Biochem 81(1):145-166

9. Bergmann JH, Spector DL (2014) Long non-coding RNAs: modulators of nuclear structure and function. Curr Opin Cell Biol 26:10-18

10. Barsyte-Lovejoy D, Lau SK, Boutros PC, Khosravi F, Jurisica I, Andrulis IL, Tsao MS, Penn LZ (2006) The c-Myc oncogene directly induces the H19 noncoding RNA by allele-specific binding to potentiate tumorigenesis. Cancer Res 66(10):5330-5337

11. Huarte M, Guttman M, Feldser D, Garber M, Koziol MJ, Kenzelmann-Broz D, Khalil AM, Zuk O, Amit I, Rabani M, Attardi LD, Regev A, Lander ES, Jacks T, Rinn JL (2010) A large intergenic noncoding RNA induced by p53 mediates global gene repression in the p53 response. Cell 142(3):409-419

12. Fagerberg L, Hallstrom BM, Oksvold P et al (2014) Analysis of the human tissue-specific expression by genome-wide integration of transcriptomics and antibody-based proteomics. Mol Cell Proteomics 13(2):397-406

13. Wang J, Liu X, Yan C, Liu J, Wang S, Hong Y, Gu A, Zhao P (2017) LEF1-AS1, a long-noncoding RNA, promotes malignancy in glioblastoma. Onco Targets Ther 10:4251-4260

14. Sun M, Nie F, Wang Y, Zhang Z, Hou J, He D, Xie M, Xu L, de W, Wang Z, Wang J (2016) LncRNA HOXA11-AS promotes proliferation and invasion of gastric Cancer by scaffolding the chromatin modification factors PRC2, LSD1, and DNMT1. Cancer Res 76(21):6299-6310

15. Wang Y, Wu K, Yang Z, Zhao Q, Fan D, Xu P, Nie Y, Fan D (2015) Multidrug-resistance related long non-coding RNA expression profile analysis of gastric Cancer. PLoS One 10(8):e0135461

16. Kong H, Wu Y, Zhu M, Zhai C, Qian J, Gao X, Wang S, Hou Y, Lu $\mathrm{S}$, Zhu H (2016) Long non-coding RNAs: novel prognostic biomarkers for liver metastases in patients with early stage colorectal cancer. Oncotarget 7(31):50428-50436

17. Schmitt AM, Chang HY (2016) Long noncoding RNAs in Cancer pathways. Cancer Cell 29(4):452-463

18. Liu XH, Sun M, Nie FQ, Ge YB, Zhang EB, Yin DD, Kong R, Xia R, Lu KH, Li JH, de W, Wang KM, Wang ZX (2014) Lnc RNA HOTAIR functions as a competing endogenous RNA to regulate HER2 expression by sponging miR-331-3p in gastric cancer. Mol Cancer 13:92

19. Cheng Z, Guo J, Chen L, Luo N, Yang W, Qu X (2015) A long noncoding RNA AB073614 promotes tumorigenesis and predicts poor prognosis in ovarian cancer. Oncotarget 6(28):25381-25389

20. Wang Y, Wang Y, Li J, Zhang Y, Yin H, Han B (2015) CRNDE, a long-noncoding RNA, promotes glioma cell growth and invasion through mTOR signaling. Cancer Lett 367(2):122-128

21. Bolha L, Ravnik-Glavac M, Glavac D (2017) Long noncoding RNAs as biomarkers in Cancer. Dis Markers 2017:7243968

22. Cabili MN, Trapnell C, Goff L, Koziol M, Tazon-Vega B, Regev A, Rinn JL (2011) Integrative annotation of human large intergenic noncoding RNAs reveals global properties and specific subclasses. Genes Dev 25(18): 1915-1927

23. Yanaka Y, Muramatsu T, Uetake H, Kozaki K, Inazawa J (2015) miR-544a induces epithelial-mesenchymal transition through the activation of WNT signaling pathway in gastric cancer. Carcinogenesis 36(11):1363-1371

24. Xing T, Du L, Zhuang X, Zhang L, Hao J, Wang J (2017) Upregulation of microRNA-206 induces apoptosis of vascular smooth muscle cells and decreases risk of atherosclerosis through modulating FOXP1. Exp Ther Med 14(5):4097-4103

25. Haga CL, Velagapudi SP, Strivelli JR, Yang WY, Disney MD, Phinney DG (2015) Small molecule inhibition of miR-544 biogenesis disrupts adaptive responses to hypoxia by modulating ATMmTOR signaling. ACS Chem Biol 10(10):2267-2276

26. Arun G, Diermeier SD, Spector DL (2018) Therapeutic targeting of long non-coding RNAs in Cancer. Trends Mol Med 24(3):257-277

27. Park SM, Choi EY, Bae DH, Sohn HA, Kim SY, Kim YJ (2018) The LncRNA EPEL promotes lung Cancer cell proliferation through E2F target activation. Cell Physiol Biochem 45(3):1270-1283 